\title{
DÜBLIN
}

Technological University Dublin

ARROW@TU Dublin

2004-3

\section{Wireless Spatio-semantic Transactions on Multimedia Datasets}

James Carswell

Technological University Dublin, james.carswell@tudublin.ie

Keith Gardiner

Technological University Dublin, keith.gardiner@tudublin.ie

Marco Neumann

Technological University Dublin, marco.neumann@tudublin.ie

Follow this and additional works at: https://arrow.tudublin.ie/dmccon

Part of the Computer Sciences Commons

\section{Recommended Citation}

Carswell, J., Gardiner, K., \& Neumann, M. (2004) Wireless spatio-semantic transactions on multimedia datasets. 19th Annual ACM International Symposium on Applied Computing (ACMSAC2004) ACM Press. Nicosia, Cyprus. 14 -17 March .

This Conference Paper is brought to you for free and open access by the Digital Media Centre at ARROW@TU Dublin. It has been accepted for inclusion in Conference papers by an authorized administrator of ARROW@TU Dublin. For more information, please contact arrow.admin@tudublin.ie, aisling.coyne@tudublin.ie, gerard.connolly@tudublin.ie.

Funder: Enterprise Ireland through the Informatics

Programme 2001 on Digital Media

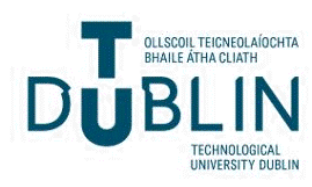


Dublin Institute of Technology

ARROW@DIT

Articles

Digital Media Centre

2004-03-01

\section{Wireless spatio-semantic transactions on multimedia datasets}

James D. Carswell

Dublin Institute of Technology, jcarswell@dit.ie

Keith Gardiner

Dublin Institute of Technology, keith.gardiner@dit.ie

Marco Neumann

Dublin Institute of Technology, marco.neumann@dit.ie

\section{Recommended Citation}

Carswell, James D. and Gardiner, Keith and Neumann, Marco: Wireless spatio-semantic transactions on multimedia datasets. 19th Annual ACM International Symposium on Applied Computing (ACMSAC2004); ACM Press; Nicosia, Cyprus; March, 2004.

This Conference Paper is brought to you for free and open access by the Digital Media Centre at ARROW@DIT. It has been accepted for inclusion in Articles by an authorized administrator of ARROW@DIT. For more information, please contact yvonne.desmond@dit.ie, arrow.admin@dit.ie. 


\section{Wireless Spatio-Semantic Transactions on Multimedia Datasets}

\author{
Dr. James D. Carswell \\ Digital Media Centre \\ Dublin Institute of Technology \\ Ireland \\ 353-1-4023264 \\ jcarswell@dit.ie
}

\author{
Keith Gardiner \\ Digital Media Centre \\ Dublin Institute of Technology \\ Ireland \\ 353-1-4023270 \\ keith.gardiner@dit.ie
}

\author{
Marco Neumann \\ Digital Media Centre \\ Dublin Institute of Technology \\ Ireland \\ 353-1-4023270 \\ marco.neumann@dit.ie
}

\begin{abstract}
Advances in spatially enabled semantic computing can provide situation aware assistance for mobile users. This intelligent and context-aware technology presents the right information at the right time, place and situation by exploiting semantically referenced data for knowledge discovery. The system takes advantage of new metadata standards to enable semantic, user, and device adapted transactions on multimedia datasets. Information accessed in the past and the activities planned by the user, the situation dependencies (e.g. location) of these activities are used to infer future information requirements. The focus of this paper describes an application of the above functionalities for performing mobile context-aware queries and updating of a multimedia spatial database of cultural heritage artifacts concerning early 20th century Dublin. It aims to exploit current consumer trends in mobile device usage by opening new markets for the increasing number of visitors to Dublin's streets. An ongoing development of this technology, the project MoCHA (Mobile Cultural Heritage Adventures), will allow the mobile cultural heritage consumer to explore a personally tailored view of Dublin's treasured artefacts, historical events and districts in an interactive and intuitive way directly on their spatially enabled PDA.
\end{abstract}

\section{Categories and Subject Descriptors}

19th ACM Symposium on Applied Computing

Mobile Computing and Applications Track

\section{General Terms}

Design, Experimentation

\section{Keywords}

location based services, spatial data transactions, semantic queries

Permission to make digital or hard copies of all or part of this work for personal or classroom use is granted without fee provided that copies are not made or distributed for profit or commercial advantage and that copies bear this notice and the full citation on the first page. To copy otherwise, or republish, to post on servers or to redistribute to lists, requires prior specific permission and/or a fee.

SAC'04, March 14-17, 2004, Nicosia, Cyprus

Copyright 2004 ACM 1-58113-812-1/03/04...\$5.00

\section{INTRODUCTION}

Project MoCHA (Mobile Cultural Heritage Adventures) is the commercial extension of the Cultural Heritage Interfaces (CHI) system developed at the Digital Media Centre (DMC) that successfully demonstrated the appropriateness of simulated cultural heritage environments for testing the usefulness and effectiveness of mobile and location aware information tailoring [4]. The CHI project has developed a framework and a testing environment to analyse Location-Based Services (LBS) and investigates the applicability of Semantic Web [15] strategies to make user query results more situation aware and therefore relevant to the users interest. (Figure 1)

MoCHA extends this research by utilizing the interactive narrative characteristics of the $\mathrm{CHI}$ digital storytelling engine together with its directional spatio-temporal querying functionality through implementation for location-aware mobile computing devices. MoCHA endeavours to exploit current trends in mobile device usage, together with their increasing computational power and new forms of mobile network and positioning systems, by opening new markets for the mobile Cultural Heritage $(\mathrm{CH})$ consumer.

Typically, a $\mathrm{CH}$ consumer would be interested in $\mathrm{CH}$ artefacts, monuments, environments and significant historical events. Artefacts include objects that could be physically moved to an exhibition - such as texts, art, etc., while monuments include objects that remain on site, i.e. in their original environments. These include physical locations where the artefacts or monuments were collected or where they reside, or could include an historical site in itself. For example, consider a theatre where transient cultural heritage such as a play or concert took place the score / recording would be considered the $\mathrm{CH}$ artefact while the theatre would be considered the $\mathrm{CH}$ environment. Examples of historical events could include the dates/locations for the births/deaths of significant historical figures or an account of a historically significant event such as the Easter Rising in 1916 Ireland.

MoCHA defines the user context as his location in physical space and the particular hand held device (HHD) being used together with his personal preferences and profile. Consideration of which mobile device is currently being used is a primary filter for determining what data will be sent and in what format. For example, image data will not be sent to a mobile phone and video will not be sent to a PDA. 


\section{Latitude/Longitude}

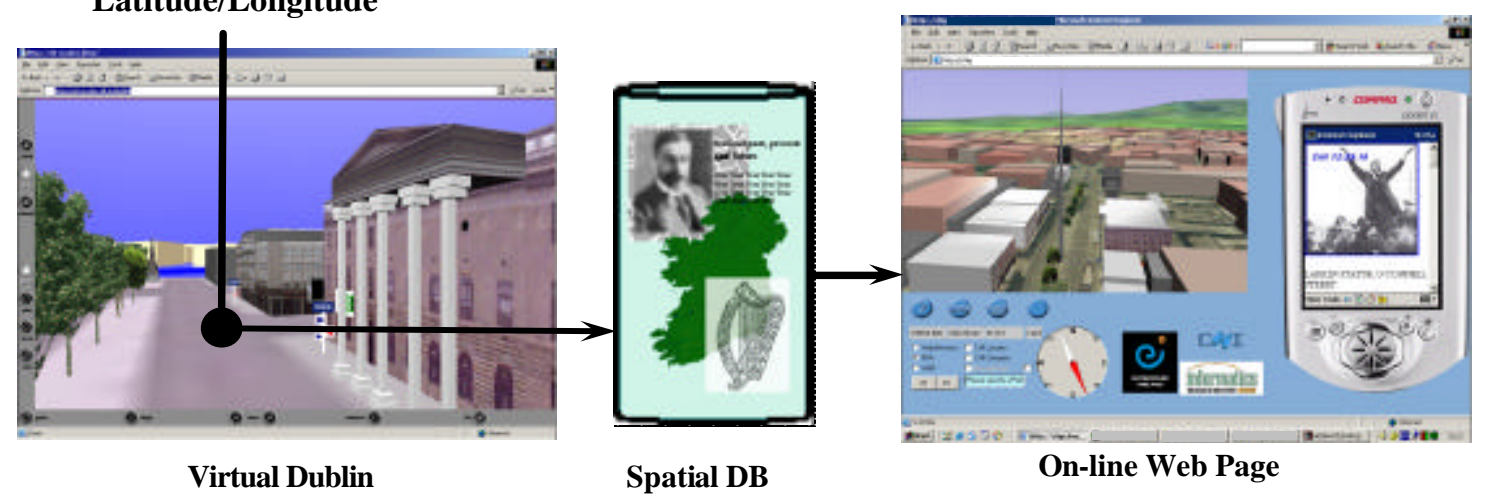

Figure 1 - The CHI Information Flow

In addition, directional view-port querying of spatial data depending on the users direct line-of-sight is taken into account for next-generation of hand-held devices (HHDs) that incorporate GPS together with digital compass capabilities. [5] Transmitting the results of such a query to HHDs requires incorporating current broadband networking technology, e.g. GPRS, UMTS and Wireless LAN computing [14].

MoCHA further extends development into coherent narrative of interactive hypermedia to include a mobile route finder to guide the user through the actual streets of Dublin. For example, if the user is looking for a particular landmark, building or $\mathrm{CH}$ event, the route finder will give exact directions on how to get there with suggestions on other places of interest based on a user's personal profile. Extrapolating this functionality to include a group of cultural sites that the user wished to visit, the application will determine how long it will take the user to reach the destinations based on, for example his current progress and also recommend the best order to visit all the sites based on the user's current position (e.g. shortest path) or any combination of criteria.

Functionality to upload personal annotation in the form of context-aware time-stamped "post-it" notes will be added to increase interactivity by further personalising the user's navigation experience for either themselves, selected user groups (e.g. friends, classmates, etc.), or indeed for all users. This particular interactivity enhancement can be exploited further by giving each user a buffer of a certain radius around his current location and if two users' are in the same buffer area they will be notified. Indeed, all members of a particular user group could be highlighted in real-time on a backdrop map display. A digital tour guide will also be added to pop up with advice on certain elements to bring an even more interactive feel to the system. This technique is used very effectively in computer games where the guide is used to expand and bridge gaps in the development of the storyline.

\section{BACKGROUND}

Dynamic websites are a familiar concept to most on-line web users where the content of the site is generated dynamically according to user demand or input. This web/hypertext paradigm, in the context of mobile users navigating the "site" spatially, in this case the actual streets of Dublin's City Centre, is the focus of MoCHA. To navigate the streets of Dublin effectively, we require spatially enabled mobile hand-held devices combined with wireless network access.

The most common application of location-based services is restricted to stationary information systems or proprietary implementations e.g. car navigation systems. These systems do not take advantage of on-demand network connections and up-todate information. To overcome these limitations, it is necessary and recommended to extend the well-proven web services of the Internet to the world of IP connected wireless mobile devices. New network architectures like 3G, GPRS, HSCSD and public W-LAN will improve the broadband for multimedia applications and expand the ubiquity of information access for mobile computing.

Project MoCHA uses standard Internet Protocol (IP) and integrates distributed system environments to reach the highest standard of services that can be implemented on today's industry devices. In combination with GPS and local positioning systems, mobile devices can act as an agent to guide users around the physical environment thus enhancing their experience of physical space. A particular aspect is the implementation of services that notify the user about relevant contextual information [7]. The real innovation of MoCHA becomes clear if we look at current user demands and the limitations of search strategies with respect to the integration of spatially enabled data into current applications - an important requirement for any user using a situation aware system.

Commercial applications of mobile computing suffer from the inability to share database content because of data format inconsistencies and/or proprietary data structures. In the last few decades, interoperability has become a critical issue (largely for facilitating automation) in many areas of $\mathrm{CH}$ information management and sharing [8]. Interoperability refers to the capability of autonomous systems to exchange, (in our case $\mathrm{CH}$ data) and to handle processing requests, by means of a common understanding and interface, across system and domain specific boundaries. This is especially true in the context of heterogeneous $\mathrm{CH}$ information that has been collected, digitised, and stored over time in many different repositories and formats resulting in datasets that are not compatible or searchable across the $\mathrm{CH}$ community.

MoCHA consequently supports the concept of separation of content data and data structure for representation, through the 
introduction of a semantic layer to improve the search for information and the maintenance of databases [11]. It incorporates the use of open standards like XML, RDF and the implementation of ontologies, catalogues and indexes for the annotation of $\mathrm{CH}$ artefacts through manual, semi-manual and automated processes to provide users with the capacity to treat multiple digital collections in various cultural institutions as one.

\section{THE MOCHA SYSTEM}

To realise a working MoCHA prototype, the main components are implemented in a three-tier web-based architecture typical of spatially enabled mobile enterprise applications [3], i.e. it comprises three layers, namely the Client Layer, the Application Server Layer, and the Database Layer.

All communications between the client layer and the database are conducted through the application server layer. The application, in our case the query building and query result formatting, is executed on the application server. The client communicates with the application server using the existing HTTP networking protocol.

\subsection{Client Layer}

The client layer consists of spatially enabled mobile devices to display spatially/semantically relevant $\mathrm{CH}$ data about Dublin's City Centre. Initially, the user's location in space combined with the particular mobile device employed determines the user's context. The purpose therefore of this implementation is to automatically push relevant data from the database layer to the client layer based on this limited contextual view. Consideration to which mobile device is being used will determine what data will be sent and in what format. In an enhancement to this initial implementation, the definition of the user's context is expanded to include user preferences and profile (e.g. the user's interests, age, task, etc.) in addition to the location, device display capabilities, and bandwidth/protocol capabilities, etc.

\subsection{Application Server Layer}

The application server environment for MoCHA is an Oracle 9iAS. This layer contains several Enterprise Java Beans (EJB's) described in the following.

The MoCHA session EJB is responsible for the communication with the client. An application server component of this type is instantiated for each user session and is responsible for monitoring changes in the user's context. For example, if the position of the user's location in space changes, this EJB will pass the new latitude/longitude coordinates to the entity beans. Other relevant context data will also be passed at this time, including the type of mobile device employed and any user profiles that may exist.

SQL statements are needed to query the spatial database for information to be displayed on the client layer. For example, when the user's context is received from the MoCHA EJB, a query is constructed and sent to the Oracle database engine for execution. The entity beans responsible for the construction, validation and execution of SQL statements are called SQL EJB's.

The difficulty in creating a narrative hypermedia system is to develop methods to mine information for different contexts.
Portions of one piece of media may need to be presented separately according to user context. For example, a user's context may include his interest in architecture, but not in politics. In this scenario, the SQL EJB builds a query in relation to architectural $\mathrm{CH}$ information about a building, but its political relevance is ignored. References to the query results are returned to the MoCHA EJB via the SQL EJB, which in turn notifies the client application of available content. If the same query is run again (i.e. no change in the user context), the MoCHA EJB reinvokes the SQL EJB that was previously instantiated thereby eliminating the need to re-interpret the query.

The middleware components format on request by the client the query results into a selection of devices, including mobile phone, and PDA. XML is used in conjunction with XSLT (extensible stylesheet language transformations) to convert XML data into the various required formats e.g. HTML, WML, etc. The advantage of using XSLT for such a scenario is that the underlying XML data structure remains the same, and by applying XSL stylesheets the data is formatted on-the-fly to whatever format is desired by using an XSLT processor.

\subsection{Semantic Layer}

The increasing number of documents on the World Wide Web has reached the limitations of conventional search approaches and new search strategies are needed. Searching for particular information in current web architectures is restricted to keyword matching and domain focused category browsing. The documents bear no explicit and standardised semantic metadata about intrinsic content. Search engines index available documents on the web in most cases by parsing the complete document for the keywords and Boolean combinations. Advanced search engines introduce techniques like Latent Semantic Indexing for natural language search engines that enable users to categorize documents from text documents [2].

Semantics in a broad sense describe the relations between things and their varying significance for the receiver. This rather wide interpretation is not addressed in current research. However, one prominent and focused attempt at a pragmatic approach is the Semantic Web representation of data on the World Wide Web based on the Resource Description Framework (RDF) [12]. RDF integrates applications using XML for syntax and Universal Resource Identifier (URI) for naming. The Semantic Web therefore extents the current web to give information a welldefined meaning and better enable computers and people to work in cooperation [1].

The introduction of a semantic layer within MoCHA is the key to generating higher-value information query results. Based on spatial filtering processes, the system can sort, organize and restructure the available information to meet the need of the user in the current situation. MoCHA implements a mixture of dynamic ontology driven geographical information systems, catalogues, index based structures and an accumulator and information recommender system. The system stores information in the central cultural heritage database and keeps user information in short term memory [11].

\subsubsection{MoCHA Semantic Query Scenario}

While the user navigates the MoCHA system the client layer dispatches a query to the EJB middleware. The documents in a selected area are passed on to the semantic interpreter to 
determine the conceptual environment. The user's agent (i.e. the client) evaluates the semantic property and compares the conceptual environment of the document(s). The result is compared to the agent's conceptual definition to satisfy the initial search context. However, in order for ontologies to be shared, they must be congruent with other shared ontologies, otherwise they have to be compared and integrated; yet another active ontology research topic [17].

The Semantic Web goes beyond these limitations and introduces a predefined semantic markup for web resources. The semantics are encoded in RDF statement triples, consisting of Resource, Property and Value - sometimes termed 'subject', 'predicate' and 'object' to describe a particular relationship. Semantics encoded into RDF triples can not only be used by human readers but also processed by machines. RDF therefore is mainly a mechanism to represent resources and their description in a direct-labelled graph.

\subsubsection{Ontology Description and RDF Schema}

To improve the information retrieval process and provide users of MoCHA with more relevant information about available data resources, the RDF metadata has to be related to the MoCHA domain ontology, which is itself implemented into a RDF Schema.

The query process for semantic evaluation of RDF descriptions is implemented on the Application Server session EJB and utilises the Jena Java API for RDF [10] to generate the model graph. For the purpose of the initial implementation of semantic exploitation, the MoCHA ontology only defines relationships between content documents stored in the Oracle Spatial database. Each content document can be accessed with a unique URL, which automatically adapts the database documents into a XML device independent tree structure and finally applies a XSLT style sheet conversion to suit the mobile device requirements for display.

The introduction of RDF metadata allows MoCHA to locate, through querying RDF statements with the RDQL query language, conceptually similar documents and selects only the spatially nearest related document for immediate display. Additionally the user can take tangents and traverse the graph manually with the help of embedded hyperlinks in the cultural heritage document. The curator of the cultural heritage content has the option to annotate the $\mathrm{CH}$ data with time properties to allow for the introduction of a narrative structuring of possible $\mathrm{CH}$ presentations that result in predefined "guided" tours. The spatial database therefore guides the user from one cultural heritage location to another with geographic direction: e.g. "go NE $300 m$ " iteratively refined until the user has reached the next point of interest.

\subsection{Database Layer}

The database implemented for Project MoCHA is Oracle 9i Spatial, which provides spatial object type storage, extended SQL access, spatial operations, and indexing as well as map projections and coordinate systems support [13]. Through this functionality, spatial queries are efficiently executed without the additional overhead of maintaining coordinate information separate from the attribute data. This is accomplished by defining the attribute information and a spatial data type (which implies associated coordinate data), e.g. either a point, line, or area. The primary advantage of spatial data types is that SQL queries can be restricted to a pre-defined geographical area, e.g. within a $10 \mathrm{~m}$ radius of a given location. By exploiting the spatial indexing mechanisms inherent to Oracle 9i, which essentially organises the information within the database tables according to their geographic location, all location relevant data can be retrieved efficiently.

Navigation through the $\mathrm{CH}$ data can begin and end at specific times and places and pass specific landmarks along the way. As each of the landmarks is encountered in turn, a particular "story object" will be retrieved about its historic/touristic/CH significance. It is the text of this "story" that comprises the bulk of the data stored in the MoCHA database layer.

The position of the viewer combined with the direction of the line-of-sight is used to develop a 2D viewer-based directional query processor that utilises an oriented, bounded object together with standard Oracle Spatial topological and metric operations to query the database. Our approach uses the line-of-sight direction vector to represent orientation and constructs a view-port of varying, user-defined dimensions as the primary filter when querying the database. As the direction of the user changes, the view-port is reconstructed in real time to reflect the users new line-of-sight search space. This method of querying the database is similar to a standard range query except that the shape of the view-port window is user defined and has orientation. This approach does not include new indexing data structures or access methods, but instead utilises the already well-known R-tree index data-structure to perform the spatial queries [9].

The MoCHA prototype has been implemented on a Windows NT platform with a particular focus to the spatial query generation on the middle tier of the system [5]. A simple experiment illustrates the advantage of the view-port directional query compared to the range query (non-directed window query) $[6,16]$.

Figure 2(a) illustrates how the CHI spatial database was organised previously and how the data was queried using a range query. Objects A, B, C and D represent street building blocks in the system. The points surrounded by circles represent $\mathrm{CH}$ artefacts. From the points a buffer is constructed and inserted into the spatial database table as a Spatial Data Type (SDT) object. If the Query window interacts with any of the buffers, the resulting data set is extracted from the database and presented to the user. A problem with the range query is as follows: if the query window is situated in Data Area 3 and the orientation of the user is northwest the returned data is data point 3 , when the actual data should be data point 1 . Similarly if the user is facing east the returned data is Data 3 when it should be Data 4.

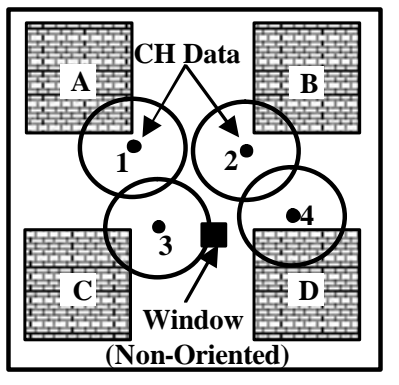

(a)

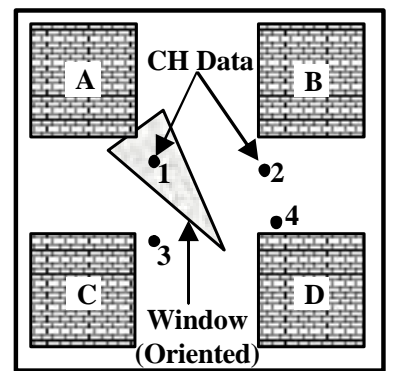

(b)
Figure 2: Comparison of Query Methods 
The improved query approach is illustrated in Figure 2(b). In this example the query window is an oriented and dynamically generated triangle based on the users position. In addition to the buffer around the $\mathrm{CH}$ point the new query can be used to maximise accuracy of view port intersections by querying exact $\mathrm{CH}$ artefact and monument positions as illustrated in 2(b). If the user's viewpoint is situated in the same position as in the previous example and is also facing northwest, the data that is returned is data point 1 together with building $\mathrm{A}$, while an orientation change to the east results in data point 4 intersection and building $\mathrm{D}$ intersection.

\section{CONCLUSIONS}

MoCHA is an on-going development that adds a mobile spatiosemantic component to web-based context-aware navigation of multimedia datasets. It aims to exploit current trends in mobile device usage by opening new markets for the increasing number of visitors to Dublin's streets every week. MoCHA allows the mobile $\mathrm{CH}$ consumer to explore a personally tailored view of Dublin's treasured artefacts, historical events and districts in an interactive and intuitive way directly on their spatially enabled PDA. To strengthen MoCHA's overall commercial appeal, mobile route finders help guide users through Dublin's $\mathrm{CH}$ environment and leave virtual context-aware "post-it" notes to further enhance the interactive nature of mobile $\mathrm{CH}$ adventures.

\section{ACKNOWLEDGMENTS}

Support for this research from Enterprise Ireland through the Informatics Programme 2001 on Digital Media is gratefully acknowledged.

\section{REFERENCES}

[1] Berners-Lee, T., Hendler, J. and Lassila, O. The Semantic Web. Scientific American 184(5), 34-43, 2001.

[2] Berry, M.W., Dumais, S.T., Shippy,A.T. A Case Study of Latent Semantic Indexing, January 1995.

[3] Bertolotto, M., Carswell, J. D., McGeown, L., and McMahon, J. iSmart+iSIS: Deploying Integrated Web-Based Spatial Applications Within an Oracle Database Environment, International Workshop on Web Geographical Information Systems (WGIS2001), IEEE CS Press, Kyoto, Japan, 2001.

[4] Carswell, J. D., Eustace, A., Gardiner, K., Kilfeather, E., and Neumann, M. An Environment for Mobile Context-Based Hypermedia Retrieval. 13th International Conference on Database and Expert Systems Applications (DEXA2002), IEEE CS Press, Aix en Provence, France, September 2002, 532-536.
[5] Gardiner, K., Carswell, J. D. Viewer-Based Directional Querying for Mobile Applications, Third International Workshop on Web and Wireless Geographical Information Systems (W2GIS2003), IEEE CS Press, Rome, Italy, 2003.

[6] Guting, R.H. An Introduction to Spatial Database Systems, VLDB, 3:357-399, 1994.

[7] Hinze, A. Voisard, A. Location- and Time-based Information Delivery in Tourism, Advances in Spatial and Temporal Databases, 8th International Symposium, SSTD 2003. Springer Verlag Lecture Notes in Computer Science. Vol. 2750. Santorini Island, Greece, July 24 - 27, 2003.

[8] International Organization for Standardization (ISO). Information and documentation -- A reference ontology for the interchange of cultural heritage information, ISO/CD 21127, 2002. URL: http://www.niso.org/international/SC4/n500.pdf

[9] Liu X., Shekhar, S., Chawla, S. Object-Based Directional Query Processing in Spatial Databases, TKDE 15(2), 295 304, 2003.

[10] McBride, B. Jena: A Semantic Web Toolkit, HewlettPackard Laboratories, Bristol, UK. IEEE INTERNET COMPUTING, November/December 2002, 55-59

[11] Neumann, M. Spatially Navigating the Semantic Web for User Adapted Presentations of Cultural Heritage Information in Mobile Environments, 1st International Workshop on Semantic Web and Databases (SWDB'03), Berlin, 2003.

[12] Resource Description Framework (RDF) Model and Syntax Specification, 1999, URL: http://www.w3.org/TR/1999/REC-rdf-syntax19990222/

[13] Rigaux, P., Scholl, M., and Voisard, A. Spatial Databases, Academic Press, Morgan Kaufmann Publishers, 352-360, 2002.

[14] Schiller, J. Mobile Communications, Addison-Wesley, 83127;161-213, 2000.

[15] Semantic Web, World Wide Web Consortium (W3C), URL: http://www.w3.org/2001/sw/

[16] Shekhar, S., and Chawla, S. Spatial Databases: A Tour, Prentice Hall Publishers, 2003.

[17] Wache,H., Vögele, T., Visser,U., Stuckenschmidt, H., Schuster,G., Neumann, H., Hübner S. Ontology-Based Information Integration: A Survey, The BUSTER Project, Intelligent Systems Group, Center for Computing Technologies, University of Bremen, 2001. 